\title{
NEUTRONIC MODELLING OF THE FFTF CONTROL ROD WORTH MEASUREMENTS WITH DIFFUSION CODES
}

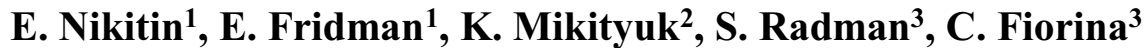 \\ ${ }^{1}$ Helmholtz-Zentrum Dresden-Rossendorf (HZDR), \\ Bautzner Landstraße 400, DE-01328 Dresden, Germany \\ ${ }^{2}$ Paul Scherrer Institute (PSI), \\ $\mathrm{CH}-5232$ Villigen-PSI, Switzerland \\ ${ }^{3}$ École Polytechnique Fédérale de Lausanne (EPFL), \\ $\mathrm{CH}-1015$ Lausanne, Switzerland \\ e.nikitin@hzdr.de, e.fridman@hzdr.de, konstantin.mikityuk@psi.ch, \\ stefan.radman@epfl.ch,carlo.fiorina@epfl.ch
}

\begin{abstract}
This paper presents an assessment of three deterministic core simulators with the focus on the neutronic performance in steady-state calculations of small Sodium cooled Fast Reactor cores. The selected codes are DYN3D, PARCS and the novel multi-physics solver GeNFoam. By using these codes, the multi-group diffusion solutions are obtained for the selected twenty control rod worth measurements performed during the isothermal physics tests of the Fast Flux Test Facility (FFTF). The identical set of homogenized few-group cross sections applied in the calculations is generated with the Serpent Monte Carlo code. The numerical results are compared with each other as well as with the measured values. The obtained numerical results, such as the multiplication factors and control rod worth values, are in good agreement as compared to the experimental data. Furthermore, a comparison of the radial power distributions is presented between DYN3D, PARCS and GeN-Foam. Ultimately, the power distributions are compared to the full core Serpent solution, demonstrating an adequate performance of the selected deterministic tools. In overall, this study presents a verification and validation of the neutronic solvers applied by DYN3D, PARCS and GeN-Foam to steady-state calculations of SFR cores.
\end{abstract}

KEYWORDS: SFR, DYN3D, PARCS, GeN-Foam, diffusion solver

\section{INTRODUCTION}

This paper presents a study to verify and validate three neutron diffusion solvers for steady-state calculations of small Sodium cooled Fast Reactor (SFR) cores. These solvers are part of the foundation of three reactor core simulators: DYN3D [1], PARCS [2] and GeN-Foam [3].

The diffusion solvers of DYN3D are based on the nodal expansion methods (NEM). The ones that are suitable for SFR calculations, as they are derived for hexagonal-z geometry, form the family of HEXNEM solvers. These solve the two-dimensional equations of hexagonal geometry in radial planes and connect 
with the axial one-dimensional equation by transverse integration. The hexagonal solver of PARCS is also based on NEM, however in contrast to HEXNEM, it divides the hexagons into six triangles and then employs polynomial expansion of the fluxes for each triangular node. This solver is called the Trianglebased Polynomial Expansion Nodal (TPEN) method. The diffusion solver of GeN-Foam is based on a finite-volume discretization with arbitrary unstructured meshes. In this work, an extruded mesh had been employed and each assembly has been radially subdivided into 54 triangles.

For code assessment, a selection of the isothermal physics tests [4], [5] performed at Fast Flux Test Facility (FFTF) was modeled in frames of IAEA Coordinated Research Project on Benchmark Analysis of FFTF Loss of Flow Without Scram Test. Twenty control rod worth measurements were simulated with the selected codes for validation purposes. The fully loaded critical core configuration was modeled to verify the selected solvers for correct prediction of the radial power distribution. In the code-to-code comparison, the reference full core solution was generated with Serpent [6] Monte Carlo code.

The following section provides the description of the benchmark, i.e. the reference core description, the routine used to generate homogenized multi-group cross sections for diffusion calculations of SFR cores, and a summary of selected tests for this study. In Section 3, the numerical results of DYN3D, PARCS and GeN-Foam are presented and compared to the benchmark experiment as well as to each other. Section 4 summarizes this paper.

\section{DESCRIPTION OF THE BENCHMARK}

The code benchmarking was performed on a $298.45 \mathrm{~cm}$ high and $230 \mathrm{~cm}$ wide section (Fig. 1) of the fully loaded FFTF core at beginning-of-life, as described in [5]. The short benchmark description provided here is adopted from the preceding study of the authors [7].

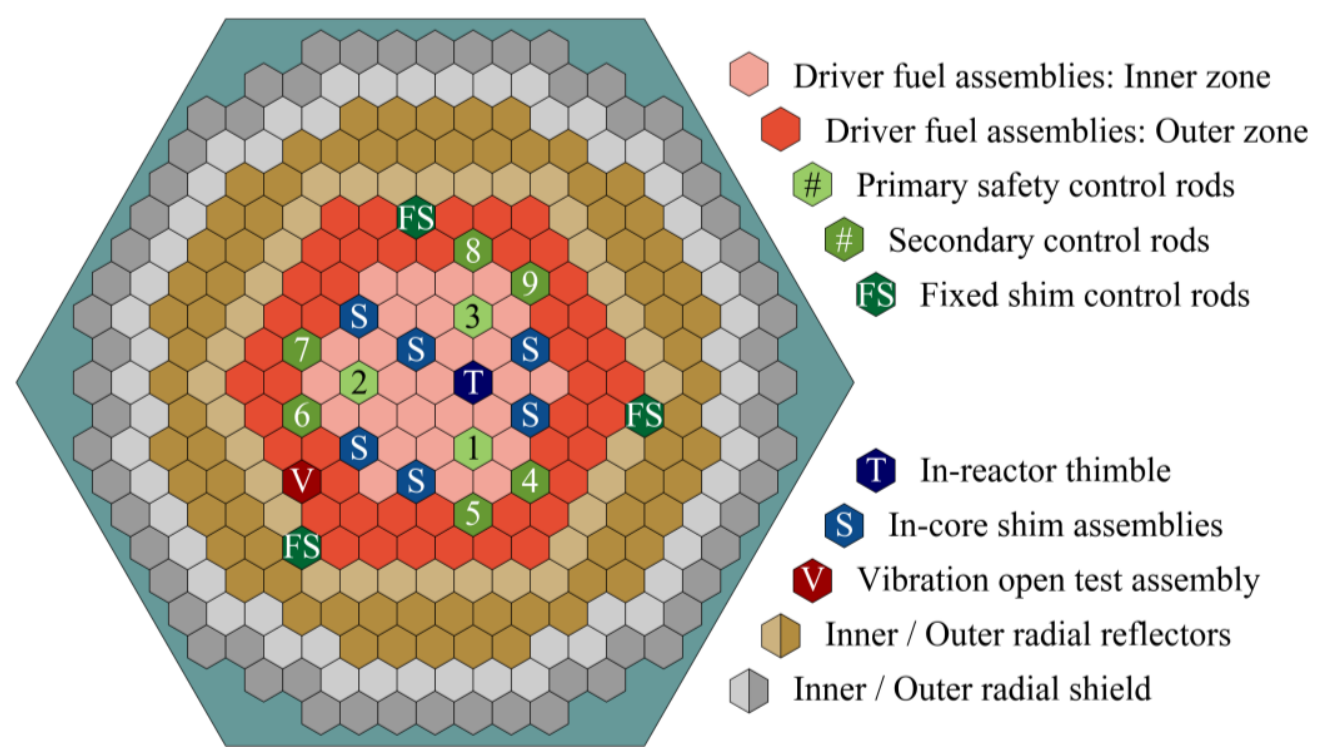

Figure 1. References core: radial core layout. Adapted from [5].

\subsection{Core Description}

The FFTF has a 400 MWth core (Fig. 1) loaded with 73 U-Pu mixed oxide (MOX) driver fuel assemblies. The core is divided into an inner and outer zone with different $\mathrm{Pu}$ content of 22.43 and 27.37 wt.\%, 
respectively. Radially, the active core is surrounded by 106 reflector and additional 114 shielding assemblies. The inner core contains six in-core shim assemblies filled with steel rods instead of fuel, in order to compensate excess reactivity at the initial start-up. The in-reactor thimble and the vibration open test assembly, depicted in Fig. 1, are non-fuel assemblies filled with instrumentations for core monitoring. Twelve identical absorber assemblies with three different roles controlled the reactor: three primary assemblies for safety, six secondary assemblies for control, and three absorber assemblies fixed in fully inserted state at the active core periphery. The whole core is surrounded by sodium coolant. The experiments were conducted at zero power while the core was maintained at temperature of around $478 \mathrm{~K}$ $\left(400^{\circ} \mathrm{F}\right)$. At this isothermal state, the assembly pitch size was $12.051 \mathrm{~cm}$.

In the reactor core, a detailed structure is only available for the pin region of fuel and absorber assemblies. The rest of the core structure are homogenized by regions. Such and other simplifications were applied in the creation of the benchmark core model, because the publicly available information was either limited, or that the modeling bias originated from the simplification is negligible as compared to the total benchmark uncertainty. A very detailed discussion of the applied simplification is available in the evaluation report of the measurements [5] along with the more detailed specification of the benchmark model (dimensions, material compositions, etc.).

\subsection{Homogenized Multi-Group Cross Sections}

The homogenized cross sections (XS) were generated with Serpent Monte Carlo code [6] based on the ENDF/B-VII.0 nuclear library [8]. The XS were obtained with the following models:

- The XS for the active part (MOX) of the driver fuel assemblies were calculated using a 3D model containing a cluster of fuel assemblies from both the inner and outer enrichment zones (Fig. 2). The assemblies are modeled in full height $(298.45 \mathrm{~cm})$, i.e. including the non-fuel regions above and below the active part (e.g., shield, reflector, plenum, etc). However, the XS are generated for the fuel regions only. The radial boundary of the XS model is defined by the edge of a hexagon that has a flat-to-flat distance of $24.102 \mathrm{~cm}$. This size corresponds to double assembly pitch distance, as seen in Fig. 2. The applied boundary condition was reflective in the radial direction and black in the axial direction.

- The XS for all other regions were generated using 2D super-cell models and were corrected with the Superhomogenization (SPH) method [9], [10]. The SPH correction procedure using Serpent/DYN3D codes on 2D super-cell models was previously described by the authors [11].

- The XS were generated on the 24-group energy structure proposed by [12].

A more detailed description of the XS generation methodology as well as the justification of it's application in this study is available in preceding publications of the authors [7], [12], [13].

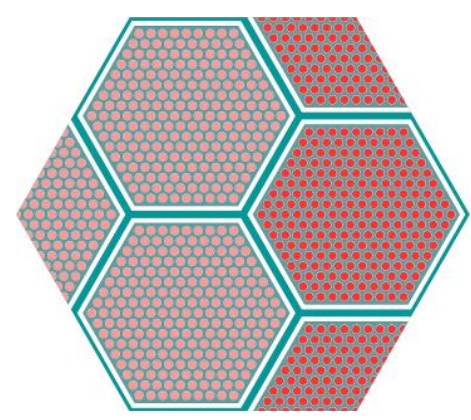

Figure 2. Radial layout of the fuel XS model (left - inner zone, right - outer zone). 


\subsection{Selected Tests}

The tests, selected for this study, were part of zero power physics tests performed on the fully loaded FFTF core in February 9, 1980. Twenty cases of control rod worth measurements were chosen for the assessments of the diffusion codes: DYN3D, PARCS and GeN-Foam. The rod worths were evaluated in respect to the nine reference states $(R 1-R 8)$ defined by the control rod positions summarized in Table I. The reactivity states of $R 1-R 8$ correspond to about 32 to $64 \mathrm{pcm}^{*}$ subcritical.

Table I. Safety and control rod positions $(\mathrm{cm})$ for reference states [5].

\begin{tabular}{|c|c|c|c|c|c|c|c|c|c|}
\hline Rod \# & $R 0$ & $R 1$ & $R 2$ & $R 3$ & $R 4$ & $R 5$ & $R 6$ & $R 7$ & $R 8$ \\
\hline 1 & 91.440 & 91.440 & 91.440 & 91.440 & 91.440 & 91.440 & 91.440 & 91.440 & 91.440 \\
\hline 2 & 91.440 & 91.440 & 91.440 & 91.440 & 91.440 & 91.440 & 91.440 & 91.440 & 91.440 \\
\hline 3 & 91.440 & 91.440 & 91.440 & 91.440 & 91.440 & 91.440 & 91.440 & 91.440 & 91.440 \\
\hline 4 & 36.116 & 0.000 & 35.306 & 91.440 & 24.384 & 26.416 & 25.400 & 27.686 & 25.654 \\
\hline 5 & 35.560 & 91.440 & 35.306 & 24.638 & 91.440 & 26.416 & 25.400 & 27.686 & 25.654 \\
\hline 6 & 35.560 & 0.000 & 35.306 & 24.638 & 24.384 & 91.440 & 25.400 & 27.686 & 25.654 \\
\hline 7 & 35.560 & 71.120 & 35.306 & 24.638 & 24.384 & 26.416 & 91.440 & 27.686 & 25.654 \\
\hline 8 & 35.560 & 0.000 & 35.306 & 24.638 & 24.384 & 26.416 & 25.400 & 91.440 & 25.654 \\
\hline 9 & 35.560 & 0.000 & 35.306 & 24.638 & 24.384 & 26.416 & 25.146 & 27.686 & 91.440 \\
\hline
\end{tabular}

The rod worth values were obtained by dropping individual or group of control rods from the reference subcritical states. The measured rods and corresponding reference states are summarized in Table II.

Table II. Control rod worth tests: measured rods and corresponding reference states.

\begin{tabular}{|c|c|c|c|c|c|}
\hline Case \# & Reference & Inserted $\operatorname{rod}(\mathrm{s})$ & Case \# & Reference & Inserted $\operatorname{rod}(\mathrm{s})$ \\
\hline 1 & $R 1$ & 1 & 12 & $R 2$ & 1 \\
\hline 2 & $R l$ & 2 & 13 & $R 2$ & 2 \\
\hline 3 & $R 1$ & 3 & 14 & $R 2$ & 3 \\
\hline 4 & $R 1$ & 5 & 15 & $R 3$ & 4 \\
\hline 5 & $R 1$ & 7 & 16 & $R 4$ & 5 \\
\hline 6 & $R I$ & $1+2$ & 17 & $R 5$ & 6 \\
\hline 7 & $R 1$ & $1+3$ & 18 & $R 6$ & 7 \\
\hline 8 & $R 1$ & $1+5$ & 19 & $R 7$ & 8 \\
\hline 9 & $R 1$ & $3+5$ & 20 & $R 8$ & 9 \\
\hline 10 & $R 1$ & $1+2+5$ & & & \\
\hline 11 & $R 1$ & $2+3+5$ & & & \\
\hline
\end{tabular}

\footnotetext{
* The delayed neutron fraction equals to $318 \mathrm{pcm}$ according to the inverse kinetic rod drop analyses reported in [5].
} 
Beside the comparison to experimental data, the selected codes were also assessed in a code-to-code verification analysis. The focus was on how well the power distributions are predicted by the threedimensional solvers of the codes. The $R O$ reference state was selected as test case.

\section{NUMERICAL RESULTS}

\subsection{Control Rod Worth Calculations}

The rod worth value $(\Delta \rho)$ of one test case was estimated as the reactivity change between the rodded and reference state: $\Delta \rho=\rho_{\text {rod }}-\rho_{\text {ref }}$. In order to obtain the control rod worth values from all the test cases (Table II), 28 steady-state calculations were performed with each selected code.

The control rod worth of the first eleven cases were obtained from reference state $R 1$. In these cases, the control rods 4, 6, 8 and 9 remained completely inserted in the core. Only the remaining rods were dropped one by one or in groups, as noted in Table II. The numerical results of these tests are summarized in Fig. 3. As compared to the experiment, in all cases, the numerical solutions of DYN3D, PARCS and GeN-Foam were in a very good agreement. PARCS predicts a slightly higher rod worth values as compared to DYN3D. The Gen-Foam values are somewhat lower than the results of the nodal codes. As compared to the measured values, the results of all selected codes do not exceed the measurement uncertainty of 5-6\%.

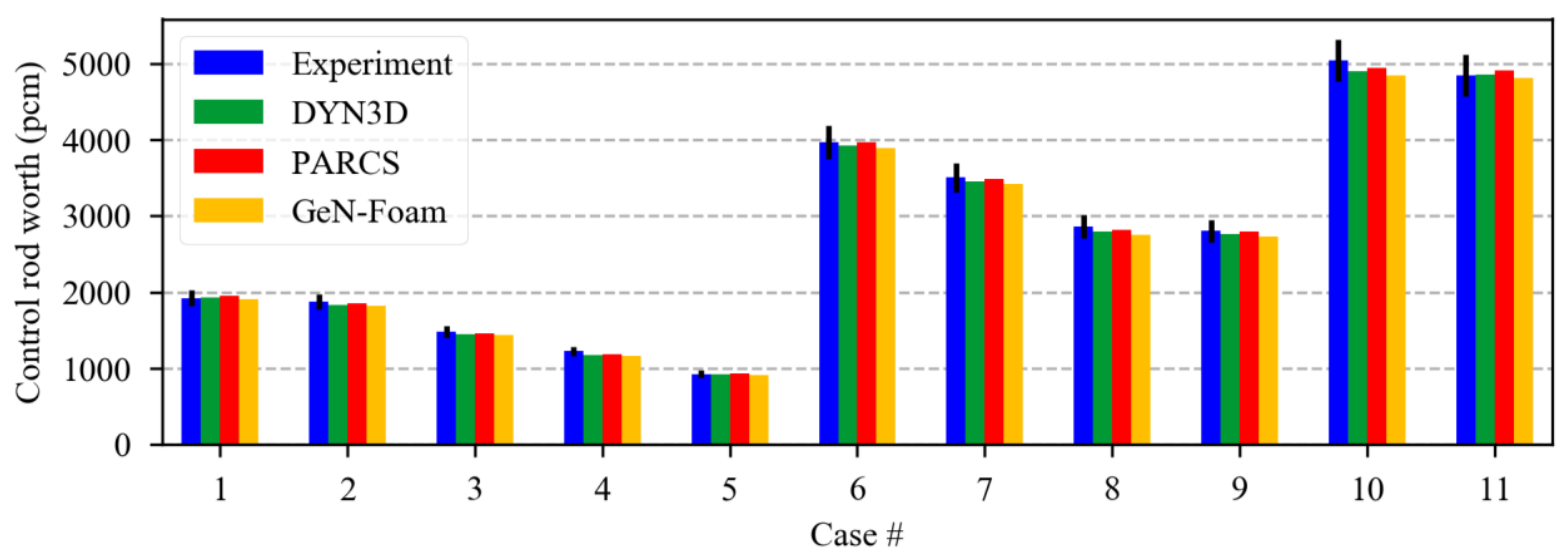

Figure 3. Comparison of rod worth values in respect to reference state $R 1$.

The numerical solutions of the individual control rod worths (case 12-20) are compared in Fig. 4. As in case 1-11, PARCS produces the highest values, GeN-Foam the lowest, while DYN3D results remain in between. The maximal range between the selected codes in these cases is around $2.9 \%$. Nevertheless, all three codes are in a very good agreement with the experiment, while the discrepancy remains below $35 \mathrm{pcm}, 27 \mathrm{pcm}$ and $50 \mathrm{pcm}$ for DYN3D, PARCS and GeN-Foam, respectively. These numerical results remain well within the measurement uncertainty band of $1 \sigma$. 


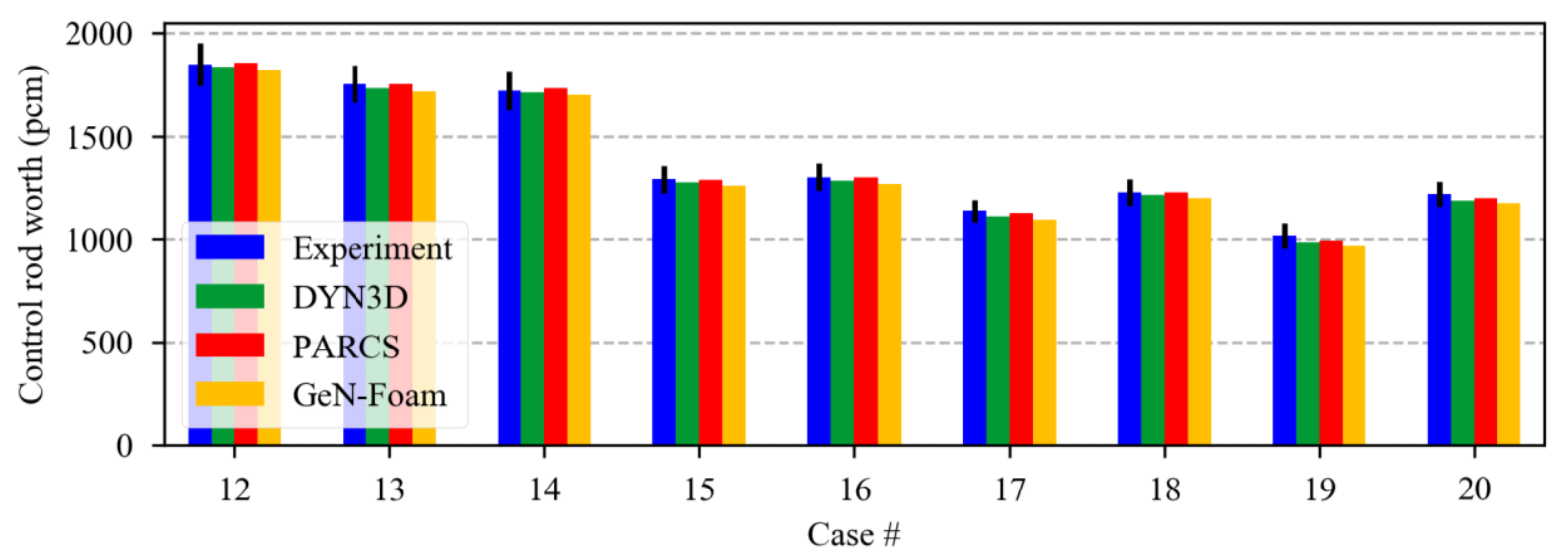

Figure 4. Comparison of individual control rod worth values.

\subsection{Code-to-Code Comparison in Reference Critical State}

The performance of the codes was also tested against each other by calculating the reference critical state ( $R 0$ in Table I). In this section, the effective multiplication factor (k-eff) and the radial power distribution was compared. As reference, the full core Monte Carlo solution was used. This was obtained by averaging the results of 20 independent Serpent calculations each of which was executed with 1500 active and 200 skipped cycles with 640000 neutron histories per cycle.

The calculated $k$-eff values are summarized in Table III. A general good agreement is observed between the diffusion codes and the Monte Carlo solution. The presented discrepancies provide a good measure of the applied diffusion solvers, because (1) identical XS sets were used in all codes and (2) both the reference full core solution and the XS were generated with Serpent. As compared to Serpent, the k-eff values of DYN3D and PARCS are lower by $151 \mathrm{pcm}$ and $239 \mathrm{pcm}$, respectively. The multiplication factor obtained with GeN-Foam is higher than the Serpent value, but remains in a very good agreement with the reference, as the difference is as small as $151 \mathrm{pcm}$.

Table III. Comparison of the effective multiplication factors of the reference critical core.

\begin{tabular}{|c|r|r|r|r|}
\hline & \multicolumn{1}{|c|}{ Serpent } & \multicolumn{1}{c|}{ DYN3D } & \multicolumn{1}{c|}{ PARCS } & \multicolumn{1}{c|}{ GeN-Foam } \\
\hline$k$-eff & $1.00284 \pm 0.00003$ & 1.00132 & 1.00044 & 1.00436 \\
\hline Difference $(\mathrm{pcm})$ & ref. & -151 & -239 & 151 \\
\hline
\end{tabular}

Although the diffusion solvers of both DYN3D and PARCS are based on the NEM, the difference in solvers provides the main source of discrepancy. However, it should be noted that the SPH correction of XS was performed with the trigonal solver of DYN3D, and the application of these XS in the PARCS solver may contribute to the observed discrepancy of $88 \mathrm{pcm}$. By applying identical meshes in both codes, the effect of different spatial discretizations was excluded in this study.

The Fig. 5 is presenting the differences in radial power distributions. As compared to Serpent, the diffusion solutions are in a very good agreement as indicated by the assembly-wise RMSD of $0.41 \%$, $0.43 \%$ and $0.74 \%$ for DYN3D, PARCS and GeN-Foam, respectively. The maximal difference is only $1.17 \%, 1.24 \%$ and $1.70 \%$, respectively. The DYN3D and PARCS results are aligned very well as the 
power difference in any assembly does not exceed $0.19 \%$. The high similarity between DYN3D and PARCS results can be explained by the fact that both diffusion solvers are based on NEM.

In case of GeN-Foam using finite-volume discretization, the map of discrepancies (Fig. 5, bottom) differs from the NEM solvers. As compared to the Serpent, GeN-Foam overestimates the local effect of control rods more than the nodal codes. That means, a lower power is predicted in the peripheral assemblies close to the fully inserted absorber rods (fixed shim control rods in Fig. 1), and higher power around the fully withdrawn control rods (control rods 1-9 in Fig. 1). The observed discrepancies between GeN-Foam and the nodal codes can be attributed to an approximate mesh convergence, and to its impact on different discretization schemes. A deeper analysis of this issue is planned for the near future. Furthermore, the fact that the SPH correction on the XS was performed with a solver different to GeN-Foam may not be neglected.
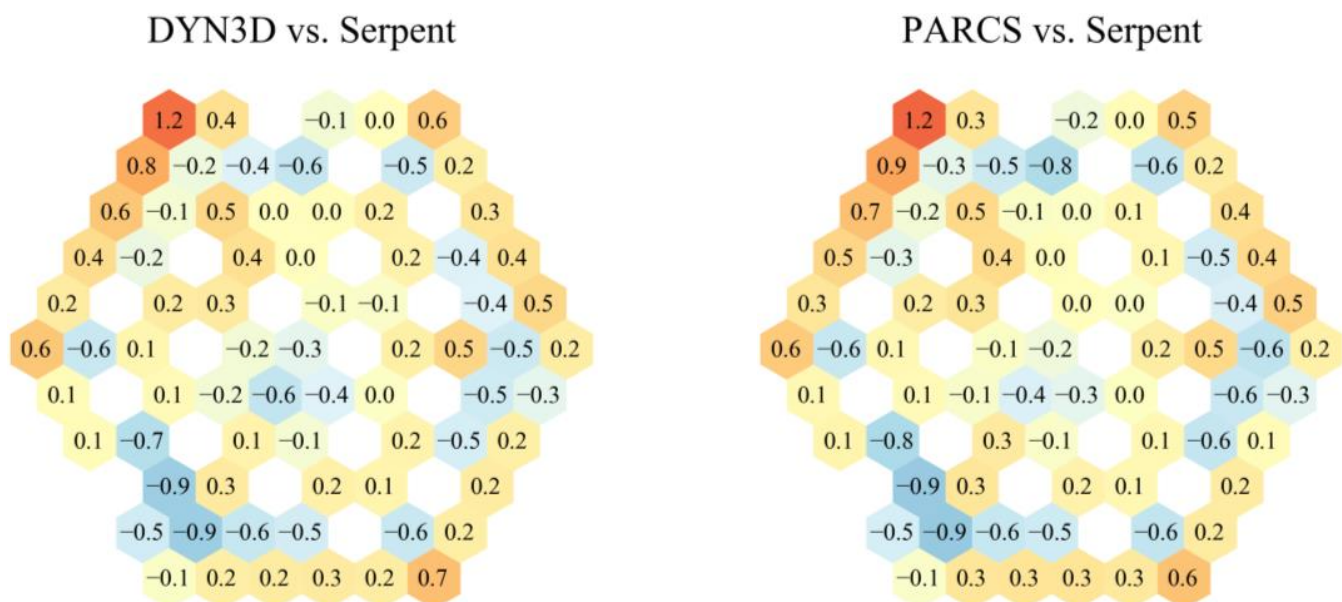

GeN-Foam vs. Serpent

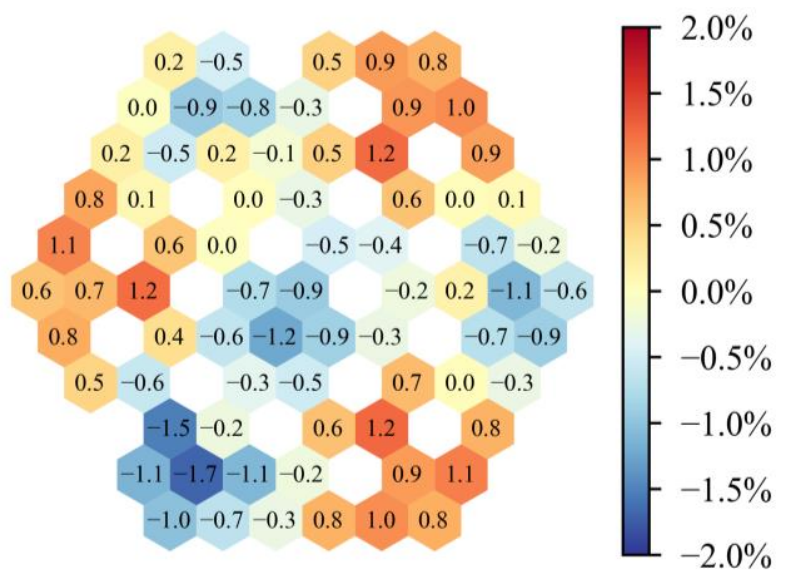

Figure 5. Difference in radial power distributions (\%), deterministic diffusion vs. Monte Carlo.

\section{SUMMARY}

A selection from the isothermal physics tests performed on the FFTF core was modeled with three different reactor core simulators: DYN3D, PARCS and GeN-Foam. In this study, the neutronic performance of the selected diffusion codes was assessed by modeling twenty cases of control rod worth 
measurements. All three codes performed very well as they predicted the experimental values within $1 \sigma$ measurement uncertainty without exception. Therefore, the presented results contribute to the validation of DYN3D, PARCS and GeN-Foam for steady-state neutronic calculations of small sized SFR cores.

Beside the control rod worth calculations, the selected codes were also compared against each other by modeling the critical core configuration of the FFTF. The results of the diffusion codes, i.e. the $k$-eff values and the radial power distributions were evaluated in respect to the reference full core Monte Carlo solution obtained with Serpent. Generally, a good agreement was observed between the codes. The radial power distributions obtained with the nodal solvers of DYN3D and PARCS were almost in perfect alignment, demonstrating similarity in their solvers. Slightly higher discrepancies were observed between GeN-Foam using finite-volume discretization and the Monte Carlo reference than in the case of the nodal diffusion codes. Nevertheless, the presented results provide additional contribution to the verification of DYN3D, PARCS and GeN-Foam for spatial neutronic analyses of SFR cores.

\section{ACKNOWLEDGMENTS}

We thank Victor Bluneau from Grenoble INP Phelma for assistance with the PARCS models.

\section{REFERENCES}

[1] U. Rohde et al., "The reactor dynamics code DYN3D - models, validation and applications," Prog. Nucl. Energy, vol. 89, pp. 170-190, 2016.

[2] T. J. Downar, Y. Xu, V. Seker, and N. Hudson, "PARCS v3.0 - U.S. NRC Core Neutronics Simulator. Theory Manual," 2010.

[3] C. Fiorina, I. Clifford, M. Aufiero, and K. Mikityuk, "GeN-Foam: a novel OpenFOAM® based multi-physics solver for 2D/3D transient analysis of nuclear reactors," Nucl. Eng. Des., vol. 294, pp. 24-37, Dec. 2015.

[4] R. B. Rothrock, "FFTF isothermal physics tests," Hanford Engineering Development Laboratory, Richland, WA, 1981.

[5] J. D. Bess, "Evaluation of the Initial Isothermal Physics Measurements at the Fast Flux Test Facility, a Prototypic Liquid Metal Fast Breeder Reactor," in International Handbook of Evaluated Reactor Physics Benchmark Experiments, 2019th ed., NEA/NSC/DOC(2006)1, FFTF-LMFRRESR-001, revision 2, OECD Nuclear Energy Agency, 2019.

[6] J. Leppänen, M. Pusa, T. Viitanen, V. Valtavirta, and T. Kaltiaisenaho, "The Serpent Monte Carlo code: Status, development and applications in 2013," Ann. Nucl. Energy, vol. 82, pp. 142-150, Sep. 2015.

[7] E. Nikitin and E. Fridman, "Modeling of the FFTF isothermal physics tests with the Serpent and DYN3D codes," Ann. Nucl. Energy, vol. 132, pp. 679-685, Oct. 2019.

[8] M. B. Chadwick et al., "ENDF/B-VII.0: Next Generation Evaluated Nuclear Data Library for Nuclear Science and Technology," Nucl. Data Sheets, vol. 107, no. 12, pp. 2931-3060, Dec. 2006.

[9] A. Hebert, "Consistent technique for the pin-by-pin homogenization of a pressurized water reactor assembly," Nucl. Sci. Eng., vol. 113, no. 3, pp. 227-238, 1993.

[10] A. Kavenoky, "The SPH Homogenization Method," in A Specialists' Meeting on Homogenization Methods in Reactor Physics, IAEA-TECDOC-231, 1978.

[11] E. Nikitin, E. Fridman, and K. Mikityuk, "On the use of the SPH method in nodal diffusion analyses of SFR cores," Ann. Nucl. Energy, vol. 85, pp. 544-551, Nov. 2015.

[12] E. Fridman and E. Shwageraus, "Modeling of SFR cores with Serpent-DYN3D codes sequence," Ann. Nucl. Energy, vol. 53, pp. 354-363, Mar. 2013.

[13] E. Nikitin, E. Fridman, and K. Mikityuk, "Solution of the OECD/NEA neutronic SFR benchmark with Serpent-DYN3D and Serpent-PARCS code systems," Ann. Nucl. Energy, vol. 75, pp. 492497, Jan. 2015. 